\title{
Auxiliando usuários de uma unidade de saúde a parar de fumar: relato de experiência
}

\author{
Assisting users of a health care unit to stop smoking: \\ an experience report
}

\author{
Donatela Ramos ${ }^{1}$ \\ Thais da Silva Tavares Soares ${ }^{2}$ \\ Karin Viegas ${ }^{2}$
}

\footnotetext{
${ }^{1}$ Secretaria M unicipal de SaúdedePorto Alegre. N ossa Sra. do Brasil 565, Santa Teresa. 90850-510 Porto Alegre RS. donateladourado@hotmail.com ${ }^{2}$ FaculdadedeEnfermagem, Nutrição eFisioterapia, Pontifícia Universidade Católica do Rio Grandedo Sul.
}

\begin{abstract}
Smoking is responsible for 4.9 millions of deaths every year, nationwide and worldwide. It is becoming a serious public health problem because of the damages caused to human health as regards its limitations to life quality, the incapacity to work, followed by illness and death. M ost deaths occur in developing countries. Tobaccoism control strategies need to be intensi fied and improved, especially in low income areas. Herein is presented a experience re port of tobaccoism control with cognitive behavior groups, at a health care unit in the suburbs of Porto Alegre, Rio Grande do Sul State. The inexpressive yearly number of people who quit smoking shows the great challenge to be faced. Therefore it is necessary the involvement of more professionals in such issue, mainly in the public health network.
\end{abstract}

Key words Tobacco use cessation, Smoking, Selfhelp groups, Basic $\mathrm{H}$ ealth care unit, $\mathrm{H}$ ealth centers
Resumo 0 tabagismo éresponsável por 4,9 milhões de óbitos no Brasil e no mundo, a cada ano. Tratase de um problema de saúde pública cada vez mais grave pelos prejuízos que causa à saúde humana, no que diz respeito às limitações na qualidade de vida, incapacitação para o trabalho, adoecimento e morte. A maioria dos óbitos vem ocorrendo nos países em desenvolvimento. Estratégias para o controle do tabagi smo precisam ser intensificadas equalificadas, em especial junto às populações debaixa renda. Apresenta-se aqui o relato de experiência com grupos do tipo cognitivo-comportamental para o controle do tabagismo, em uma unidade de saúde da periferia da cidade de Porto Alegre, Rio Grandedo Sul. 0 núme ro ainda pouco expressivo de pessoas que anualmente deixam de fumar nos demonstra o tamanho do desafio a ser enfrentado, sendo, portanto, necessário que mais profissionais se envolvam com a questão, em especial na rede básica de saúde pública.

Palavras-chave Abandono do uso de tabaco, Tabagismo, Grupos de auto-ajuda, Unidade Básica de Saúde, Centros de saúde 


\section{Introdução}

Tabagismo: um problema de saúde pública

Sabe-se que o tabagismo é responsável por 4,9 milhões de óbitos anuais no mundo ${ }^{1}$, sendo que 40 a $45 \%$ são por câncer, dos quais 90 a 95\% por câncer de pulmão, $75 \%$ dos óbitos ocorrem por DPOC, 20\% por doença vascular e 35\% por doença cardiovascular ${ }^{2}$. Cerca de $20 \%$ da população mundial fuma, sendo que $80 \%$ dos fumantes encontram-se nos países em desenvolvimento ${ }^{3}$.

Em 1998, foi demonstrado que, no Brasil, 30,6 milhões da população brasileira eram fumantes². A O rganização Mundial de Saúde prevê que, se o atual padrão mundial de consumo de tabaco não for revertido ( 1,1 bilhão de fumantes), o número de óbitos poderá chegar a 10 milhões no ano de 2020 , dos quais $70 \%$ ocorrerão nos países em desenvolvimento 4 .

Embora a legi slação brasileira venha setornando mais proibitiva em relação ao consumo de cigarros ou assemel hados e venha cerceando a propaganda, muitos esforços ainda precisam ser feitos para que haja mais significativa redução do consumo. Segundo Pesquisa Nacional de Saúde e Nutrição, a prevalência de fumantes acima de 15 anos no país era de $32 \%$ em 1989, sendo de $18,8 \%$ em $2003^{5}$.

O Brasil é signatário da Convenção-Quadro da Organização M undial deSaúde, em vigor desde2005, a qual visa reduzir a prevalência do consumo e a exposição ao tabaco, " [...] proteger as gerações presentes e futuras das devastadoras consequências sanitárias, sociais, ambientais e econômicas, geradas pelo consumo e pela exposição à fumaça do tabaco[... $]^{\prime 6}$. Nosso país exerceu importante papel ao presidir todas as negociações referentes ao tratado, queenvolveram 192 países. Sua eleição, por consenso, para presidir as discussões, deu-se pelo avanço no controle do tabagismo; porém, a resistência interna enfrentada foi muito grande, para que pudesse obter 0 aval do congresso nacional, devido à grande pressão da indústria do fumo no país.

O Brasil é o segundo maior produtor e exportador mundial de folhas de fumo. M ais de $90 \%$ da produção encontra-se no sul do país, sendo $50 \%$ no Rio Grande do Sul ${ }^{6}$, sendo que a cidade de Venâncio Aires, no Vale do Rio Pardo, lidera a safra de fumo. Na totalização nacional, a Região Sul é responsável por $97 \%$ da produção de fumo, estando Santa Catarina em segundo lugar, seguido pelo Paraná7. Por esta razão, o governo brasileiro comprometeu-se a auxiliar técnica e financeiramente os produtores e trabalhadores da indústria nacio- nal de fumo para que avaliem alternativas econômicas, condição prevista na Convenção-Quadro ${ }^{6}$.

No Rio Grande do Sul, estado pioneiro na legislação de controle e respeito aos direitos dos nãofumantes, em 1995, 27,4\% da população adulta era fumante regular e, desses, $69,2 \%$ desejavam parar de fumar ${ }^{8}$. A prevalência do fumo, na cidade de Pelotas, avaliada em estudo de 1995, era de $34 \%{ }^{9}$. Segundo estudo posterior realizado em Pelotas, em 2002, a eliminação total do tabagismo levariaà prevenção de $54 \%$ do câncer de esôfago, de $71 \%$ do câncer de pulmão e de $86 \%$ do câncer delaringe ${ }^{10}$.

As neoplasias pulmonares são muito frequentes em todo o mundo e no Brasil é a principal causa de mortalidade por câncer. 0 hábito do tabagismo ér responsável por mais de $80 \%$ dos casos de câncer de pulmão entrehomens epor mais de $45 \%$ entre as mulheres. Dados do M inistério da Saúde, nos últimos dez anos, mostram o crescimento da doença de $57 \%$ para os homens e $112 \%$ entre as mulheres brasileiras. São Paulo, Brasília e Porto Alegre são as cidades que possuem as maiores médias anuais de incidência de neoplasias pulmonares no Brasil ${ }^{11}$.

Fuma-se mais na Região Sul do Brasil (42\% dos habitantes) ${ }^{4}$. Em Porto Alegre, a prevalência detabagi smo chega a $34,9 \%$, sendo a cidade detentora dos maiores índices conhecidos de câncer de pulmão do país ${ }^{12}$.

Em 2003, de acordo com a Secretaria Estadual de Saúde, através de inquérito domiciliar sobre comportamentos de risco e morbidades referidas de doenças e agravos não-transmissíveis - módulo tabagismo, a prevalência, em Porto Alegre, é de $28 \%$ de fumantes do sexo masculino e $23 \%$ da população feminina. Já em 2006, de acordo com a pesquisa realizada nas capitais e no Distrito Federal, através de entrevistas telefônicas com 54 mil pessoas com mais de 18 anos, divulgada pelo Sistema de M onitoramento de Fatores de Risco e Proteção para Doenças Crônicas, em Porto Alegre, $26,3 \%$ da população masculina e $17 \%$ da população feminina são fumantes ${ }^{13}$. D ados queainda chamam atenção apontam estudantes de Porto A legre de 13 a 15 anos, dos quais $37 \%$ dos meninos e $34 \%$ das meninas, como fumantes ${ }^{14}$.

Segundo a coordenação do programa de controle do tabagismo da Secretaria Estadual de Saúde do Rio Grande do Sul, já podemos verificar uma redução significativa, nos últimos anos, do índice de fumantes graças às campanhas educativas, às imagens nas carteiras de cigarro, à proibição de publicidade na mídia, à interdição do fumo em locais fechados etodas as ações de controle da rede pública contra o tabagismo ${ }^{13}$. 
Entreosquestionamentos que se colocam como desafios para aqueles que vêm procurando trabaIhar no sentido de reduzir ou eliminar o hábito de fumar, destacam-se os seguintes:

1. Porque tantas pessoas, embora desejando parar de fumar, continuam a fazêlo, mesmo conhecendo seus malefícios sobre a saúde?

2. Como avaliar esta dependência, com vistas a uma abordagem eficaz?

3. Que método empregar no âmbito da saúde pública, para tratamento dos indivíduos que desejam parar de fumar?

$N$ ão há dúvida quanto à influência física e psicológica da dependência nicotínica sobrea dificuldadeda suspensão dessehábito. Estima-seque 24\% da população adulta, em diversos países, até mesmo no Brasil, seja dependente de nicotina ${ }^{12}$.

A partir deuma capacitação realizada em agosto de 2005, iniciou-se o planejamento de grupos para controle do tabagismo em uma unidade básica de saúde, situada na periferia de Porto Alegre, segundo o método cognitivo-comportamental proposto pelo M inistério da Saúde (M S) ${ }^{2}$.

Assim, tomando por base o respectivo referencial, decidiu-se pela realização desterelato de experiência, que tem por objetivo contribuir para a análise da assistência prestada aos usuários que desejam parar de fumar, na unidade de saúde em questão, servindo possivelmente de elemento de reflexão para experiências semelhantes.

\section{M etodologia}

Enfrentando o desafio na unidade de saúde

Durante um mês e meio, realizou-se o planejamento e a divulgação do grupo de controle de tabagismo da unidade de saúde. Saliente-se que, segundo o M inistério da Saúde, a unidadeé considerada "livre de tabaco", já que nenhum dos membros da equipe é fumante, não sendo permitido 0 uso de cigarros na área interna da unidade de saúde. 0 interesse pelos usuários foi grande e 33 pessoas se inscreveram para participar do primeiro grupo. A divulgação foi feita em grupos de sala de espera, no dia 29 de agosto de 2005 - Dia Nacional de Combate ao Fumo - através de cartazes, panfletos e pela abordagem direta aos usuários em consultas médicas, de enfermagem eduranteo atendimento dos auxiliares de enfermagem da equipe.

D eixava-se claro aos interessados que, para participar do grupo, éindispensável o firmedesejo de parar de fumar.

A metodologia proposta pelo Ministério da Saúde prevê quatro reuniões em grupo, com fre- quência semanal e, pelo menos, dois encontros de manutenção e suporte; porém, na unidade de saúde estudada, optou-se por acompanhar semanalmente, em caráter individual ou grupal, os participantes do programa, em número variável de encontros, conforme a necessidade e adesão. Além do atendimento em grupo realizado pela enfermei$\mathrm{ra}$, foram realizadas consultas individuais aos usuários, com a enfermeira ou o clínico geral vinculados ao programa, previamente agendadas, a partir de avaliação particularizada de necessidade.

A unidade de saúde realizou seis grupos, a partir de outubro de 2005 até março de 2006, sem dispor de medicamentos de suporte para auxiliar a cessação do hábito de fumar. Passou-se então a contar com gomas de mascar e adesivos de nicotina com dosagens de 7, 14 e 21 miligramas, repassados ao município pelo M inistério da Saúde. De abril a outubro de 2006, foram realizados três grupos, contando com o auxílio de terapia de reposição nicotínica.

Conforme a avaliação individual do grau de dependência, com base no teste de Fagerström, os participantes dos grupos eram encaminhados à consulta médica para a solicitação e avaliação de exames diagnósticos e eventual prescrição de medicamentos de suporte, a partir da terceira reunião do grupo, segundo preconiza o M inistério da Saúde $^{2}$. A utilização do testefoi recomendada em estudo realizado na cidade de Rio Grande (RS), em 2002, por sua simplicidade, rapidez e baixo custo de aplicação ${ }^{15}$.

Garantiu-se 0 acesso às consultas médicas e de enfermagem, mediante agendamento prévio aos usuários, sempre que necessário. 0 acompanhamento individual e semanal foi realizado pela enfermeira para avaliação do uso correto da medicação, identificação de síndrome de abstinência, reforço das orientações, suporteemocional em eventuais recaídas, reencaminhamento ao médico em casos de necessidade de ajustes de doses de medicação ou avaliação sobre o uso de antidepressivos ou outros medicamentos.

Foram criados materiais próprios para facilitar as atividades em grupo, como um álbum seriado específico com os aspectos principais tratados nas reuniões, planilhas para registro das informações relevantes durante 0 atendimento e controle de frequência aos grupos, assim como uma agenda individual para registro diário do uso ou não de cigarros e seus motivos. Utilizaram-se também cartazes de anatomia humana, de patologias respiratórias relacionadas, para facilitar a compreensão das informações apresentadas. Os materiais criados foram utilizados de modo complementar aos manuais, cartazes, panfletos e técnicas especí- 
ficas propostas pelo M inistério da Saúde. Criouse ainda um "certificado" simbólico para estímulo aos fumantes em abstinência. $\mathrm{Na}$ criação desses materiais, contou-se com a colaboração de professora e acadêmicos de enfermagem da PU CRS.

Considerando-se que a observação demonstrou que pacientes que haviam participado dos grupos podiam ter parado de fumar na quarta reunião e ter retornado ao hábito posteriormente, bem como ocorria à situação inversa, isto é, continuar fumando até a quarta reunião e ter parado posteriormente, optou-se por entrevistar os participantes de todos os grupos que preenchiam o requisito deter participado atéa quarta reunião para verificar sua a situação.

\section{Resultados}

No primeiro encontro do primeiro grupo realizado, compareceram onze pessoas dentre as 33 inscritas, permanecendo quatro até $o$ último encontro.

No total de seis grupos realizados no período de outubro de 2005 a março de 2006 (sem medicação de apoio), quatro usuários deixaram de fumar até 0 quarto encontro e cinco estavam sem fumar no levantamento realizado em abril de 2007.

A Tabela 1 mostra o número de grupos realizados no período de outubro de 2005 a março de
2006, o somatório do número de participantes presentes no primeiro e no último encontro de todos os grupos realizados no período, o número dos que deixaram de fumar até a quarta sessão e no momento do levantamento feito em abril de 2007.

A Tabela 2 mostra o número de grupos realizados no período abril de 2006 a outubro de 2006 (com medicação de apoio), o somatório do número de participantes presentes no primeiro e no último encontro de todos os grupos realizados no período, o número dos que deixaram defumar até a quarta sessão e no momento do levantamento feito em abril de 2007.

A Tabela 3 mostra o número total de grupos realizados no período de outubro de 2005 a outubro de 2006, o somatório do número de participantes presentes no primeiro e no último encontro de todos os grupos realizados no período, 0 número total dos que deixaram de fumar até a quarta sessão e no momento do levantamento feito em abril de 2007.

A partir da Tabela 1, verificamos que, no período de outubro de 2005 a março de 2006, 36,4\% pararam de fumar até a quarta sessão. Conforme registros da unidade de saúde, após a quarta sessão, mais $27,3 \%$ pararam, totalizando $63,7 \%$ de fumantes em abstinência. Quarenta e cinco por cento permaneciam sem fumar no levantamento realizado em abril de 2007.

Tabela 1. Consolidação de informações do tratamento do tabagismo sem medicação de apoio - unidade de saúde.

\begin{tabular}{|c|c|c|c|c|c|}
\hline \multirow[t]{3}{*}{ Período de atendimento } & \multirow[t]{3}{*}{ Total grupo } & \multicolumn{4}{|c|}{ Dados do atendimento ocorrido no período } \\
\hline & & \multicolumn{2}{|c|}{ Número de participantes } & \multicolumn{2}{|c|}{ Participantes sem fumar } \\
\hline & & 1’a sessão & 4⿳亠口冖 sessão & 4⿳亠口冋 sessão & abril 2007 \\
\hline out/2005 a mar/2006 & 6 & 39 & 8 & 4 & 5 \\
\hline
\end{tabular}

Fonte: Unidadede SaúdeTronco - SM S/PM PA, 2007.

Tabela 2. Consolidação de informações do tratamento do tabagismo com medicação de apoio - unidade de saúde.

\begin{tabular}{|c|c|c|c|c|c|}
\hline \multirow[t]{3}{*}{ Período de atendimento } & \multirow[t]{3}{*}{ Total grupo } & \multicolumn{4}{|c|}{ Dados do atendimento ocorrido no período } \\
\hline & & \multicolumn{2}{|c|}{ Número de participantes } & \multicolumn{2}{|c|}{ Participantes sem fumar } \\
\hline & & 1 1’ sessão & 4⿳亠口冖 sessão & 4⿳亠口冋 sessão & abril 2007 \\
\hline $\mathrm{abr} / 2006$ a out/2006 & 3 & 26 & 16 & 4 & 3 \\
\hline
\end{tabular}

Fonte: UnidadedeSaúdeTronco - SM S/PM PA, 2007. 
Tabela 3. Consolidação de informações do tratamento do tabagismo com ou sem medicação de apoio - unidade de saúde.

\begin{tabular}{cccccc}
\hline Período de atendimento & Total grupo & \multicolumn{3}{c}{ Dados do atendimento ocorrido no período } \\
\cline { 3 - 6 } & & N úmero de participantes & \multicolumn{2}{c}{ Participantes sem fumar } \\
\cline { 3 - 6 } & & 1a sessão & 4a sessão & 4a sessão & abril 2007 \\
\hline out/2005 a out/2006 & 9 & 65 & 24 & 8 & 8
\end{tabular}

Fonte: Unidade de Saúde Tronco - SM S/PM PA, 2007.

No período de abril a outubro de 2006 (Tabela 2), $25 \%$ pararam de fumar até a quarta sessão e, após a quarta sessão, mais 31,3\% pararam, totalizando $56,3 \%$ de fumantes em abstinência. No levantamento realizado em abril de 2007, apenas $18,8 \%$ permaneciam sem fumar.

A faixa etária predominante dos usuários que buscaram auxílio foi de 20 a 49 anos, coincidindo com a faixa de idade em que se concentra o maior número de fumantes no Brasil'. Segundo o Instituto Nacional de Câncer (INCA), a dependência é adquirida em média até os 19 anos. Predominou a presença de associações de comportamento, com $94,44 \%$, sobre a dependência física e psíquica, entre os participantes dos grupos, avaliada a partir do teste de Fagerström ${ }^{16}$.

Entre os usuários, participantes dos grupos realizados de outubro de 2005 a outubro de 2006, havia dezessete mulheres e setehomens. Entre esses, oito conseguiram parar de fumar até a quarta sessão, sendo sete mulheres, e cinco após este momento, isto é, durante as sessões de manutenção. Dos oito usuários que pararam de fumar até a quarta sessão, seis apresentavam dependência psíquica e faziam associações de comportamento e cinco apresentavam dependência física. 0 número de cigarros consumidos por esses usuários variou de dez a quarenta por dia, em média.

Embora o conhecimento sobre os prejuízos à saúde decorrentes do hábito de fumar, entre leigos, esteja razoavel mente difundido, havia nos grupos o impacto causado pela informação de que fumar pode causar dependência física e psíquica.

\section{Discussão}

A tendência de grande redução no número inicial de participantes dos grupos, que ocorreu nos primeiros grupos, manteve-se nos demais. Embora essetipo de redução seja previsto einclusive alertado nas capacitações a quem pretendetrabal har com esses usuários, o fato não deixa de afetar os profissionais, que esperam por uma melhor adesão. A importante redução no número de usuários que prosseguiram participando após a segunda reunião, é decorrente de que já no primeiro encontro confronta-se o real desejo que o indivíduo tem de parar de fumar, através da proposta de que marque uma data para parar até a terceira reunião. Avalia-se que os que permanecem atéo último encontro estão efetivamente motivados a parar de fumar, ainda que não consigam fazê-lo no período previsto. 0 grupo, por suas características eas propostas, faria uma espécie de "seleção natural", isto é, a motivação para parar de fumar favoreceria a permanência no grupo até a quarta reunião. É importante salientar que, entreosque permaneceram até a última reunião, sem conseguir parar de fumar, ocorreu significativa diminuição no número de cigarros consumidos por dia, o que resulta em repercussão benéfica sobre a saúde do indivíduo e dos que com ele convivem.

0 tabagismo passivo caracteriza a exposição ao fumo por não-fumantes, envolve aproximadamente $80 \%$ da população, aumenta as causas de doenças respiratórias, leva a um risco aumentado decâncer depulmão einfarto em adultos edeasma, pneumonias e otites em crianças. É a terceira maior causa de morte evitável no mundo. A OM S estima quehá cerca de dois bilhões defumantes passivos no mundo?.

Não ocorreram sintomas graves relacionados com a síndrome de abstinência ${ }^{2}$ entre os pacientes que pararam de fumar sem o auxílio de medicação de apoio. A perspectiva da utilização de medicação de apoio foi importante elemento motivador para a participação e permanência nos grupos. A força de vontade dos participantes é elemento fundamental na cessação do tabagismo, pois na compa- 
ração entre o grupo que utilizou e o que não utilizou medicação de apoio, esse último grupo apresentou o mais alto percentual de abstinência.

0 indivíduo fumante, ao deixar o hábito de fumar, não pode ser considerado ex-fumante e, sim, em abstinência do cigarro, pelas características da dependência nicotínica. A experiência aqui relatada demonstra queo período deum mês pode ser curto para avaliar-seo êxito da assistência prestada no tratamento ao tabagismo, através de grupos cognitivo-comportamentais, com ou sem 0 apoio medicamentoso.

0 percentual de indivíduos que deixaram de fumar até a quarta sessão dos grupos foi maior do que 0 encontrado em outros estudos ${ }^{2}$.

O levantamento realizado em abril de 2007, seis meses a um ano e meio, após a participação nos grupos, demonstrou um percentual de abstinência de $69,57 \%$; porém, 50\% destes tiveram recaídas e voltaram a fumar. Indivíduos que deixaram de fumar costumam enfrentar recaídas entre dois dias e três meses de abstinência ${ }^{17}$.

Palavrasfinais:

persistindo no enfrentamento do problema

Da experiência acumulada, durante o período de um ano, em uma unidade básica de saúde da periferia dePorto Alegre, foi possível verificar quegrupos do tipo cognitivo- comportamental, na rede básica, associados à terapia medicamentosa, conforme metodologia proposta pelo Ministério da Saúde, atendem ao propósito de superar obstáculos práti- $\cos ^{12}$ para a cessação do hábito de fumar, quais sejam: a falta de diagnóstico médico da dependência de nicotina, o desconforto da abstinência e o número insuficientedeserviços de apoio para abandono do fumo. Porém, o aperfeiçoamento desse método, bem como novos métodos complementares, devem ser testados na busca de melhores resultados, visando à redução do tabagismo.

0 percentual de pacientes que conseguiram deixar de fumar, a partir da metodologia utilizada de grupos do tipo cognitivo-comportamental, associada ou não à terapia de reposição nicotínica, foi de $34,78 \%$, superior ao de $3 \%$ encontrado na literatura 2 .

A proposta deauxiliar usuários da rede básica a deixar de fumar constitui-se um grande desafio, exigindo persistência, disponibilidadeecriatividade por parte dos profissionais envolvidos. Trata-se de importante estratégia para facilitar o acesso da população socialmente excluída a essa ferramenta tecnológica de trabalho que, até o momento, pelo menos em nosso meio, permanece quase restrita ao atendimento prestado em hospitais e/ ou ambulatórios especializados. Desse modo, espera-se que um maior número de profissionais da rede básica de saúde envolva-se nesse tipo de atividade.

A avaliação permanente quanto aos métodos empregados ea troca de experiências entre profissionais consistem em importantes instrumentos para o aperfeiçoamento do trabalho desenvolvido, o qual busca o engajamento nos esforços nacionaise internacionaisquevêm sen do realizados para reduzir os padrões de consumo do tabagismo e, consequentemente, a diminuição da morbimortalidade decorrentes do hábito de fumar.

\section{Colaboradores}

DD Ramos eK Viegas trabalharam na concepção, pesquisa, metodologia e redação final e TST Soares, na pesquisa, metodologia e redação final. 


\section{Referências}

1. World Health Organization. Reducing risks, promotion healthy life. The world health report 2002. Geneva: WHO; 2002.

2. Brasil. M inistério da Saúde. Secretaria Nacional de Assistência à Saúde. Instituto Nacional de Câncer. Coordenação Nacional de Controle do Tabagismo e Prevenção Primária do Câncer. Abordagem e tratamento do fumante - Consenso 2001. Rio de Janeiro: INCA; 2001.

3. Guidon GE, Boisclair D. Past, current and future trends in tobaco use [HNP Discussion Paper. Economics of Tobaco Control Paper n. 6]. Washington, D.C.: The World Bank; 2003.

4. Brasil. M inistério da Saúde. Secretaria Nacional de Assistência à Saúde. Instituto Nacional de Câncer. Coordenação Nacional de Controle do Tabagismo e Prevenção Primária de Câncer. Falando sobre Tabagismo. 3a ed. Rio de Janeiro: M inistério da Saúde; 1998.

5. Brasil. M inistério da Saúde. Secretaria de Vigilância em Saúde. Instituto Nacional de Câncer. Inquérito domiciliar sobre comportamentos de risco e morbidade referida de doenças e agravos não transmissíveis. Rio de Janeiro: M inistério da Saúde; 2004.

6. Cavalcante TM. O Brasil e a Convenção - Quadro da Organização M undial da Saúde. Secretaria Executiva da Comissão Nacional (interministerial) para Implementação da Convenção Quadro para Controle do Tabaco. [site da Internet] 2006. [acessado $2007 \mathrm{mar}$ 27]. Disponível em: www.mc.gov.br/sites/600/695/ 00002184.pdf

7. Instituto Brasileiro de Geografia e Estatística. Produção agrícola municipal 2005 - Em 2005, valor da produção agrícola foi de 13,6 bi menor. [site da Internet]. [acessado 2007 mar 27]. Disponível em: www.ibge.gov. br/home/presidencia/noticias/noticia_visualizada. php?id_noticia $=740$

8. Oliveira N eto IC. Epidemiologia do tabagismo no Rio Grande do Sul [tese]. Porto Alegre (RS): Universidade Federal do Rio Grande do Sul; 1998.

9. Menezes AM B, Rigatto M, Victora CG. Chronic bronchitis and the type of cigarette smoked. Int J Epidemiol. 1995; 24:95-99.
10. M enezes $A M B$, Horta $B L$, Oliveira ALB, Kaufmann RAC, Duquia R, Diniz A, M otta LH, Centeno MS, Estanislau G, Gomes L. Risco de câncer de pulmão, laringe e esôfago atribuível ao fumo. Rev. Saude Publica 2002; 36:129-134.

11. Gil AR. Câncer de pulmão: inimigo a ser enfrentado. Prat H osp. [periódico na Internet] 2004 mar/abr [acessado 2007 mai 28]; 6(32):[cerca de 2 p.] Disponível em: http://www.praticahospitalar.com.br/pratica\% 2032/paginas/editorial\%2032.html

12. M oreira LB, Fuchs FD, M oraes RS, Bredemeir M, Cardozo S. Prevalência de tabagismo e fatores associados em área metropolitana da região sul do Brasil. Rev. Saude Publica 1995; 29:46-51.

13. M iranda AE. Índices de fumantes entre gaúchos preocupa a Secretaria de Saúde. Governo do Estado do Rio Grande do Sul. Agência de notícias. [site na Internet] [acessado 2007 mar 27]. Disponível em: http:/ /www.estado.rs.gov.br/index.php

14. Saúde gaúcha faz ofensiva contra o tabagismo. Governo do Estado do Rio Grande do Sul. Agência de notícias. [site da Internet]. [acessado 2007 mar 27]. Disponível em: http://www.estado.rs.gov.br

15. Balbani APS, Montovani JC. Métodos para o abandono do tabagismo. Rev Bras Otorrinolaringol. 2005; 71:820-827.

16. Halty LS, Huttner MD, N etto ICO, Santos V, Martins G. Análise da utilização do Questionário de Tolerância de Fagerström (QTF) como instrumento de medida da dependência nicotínica. J Pneumol. 2002; 28:180-186.

17. Jain A. Treating nicotine addiction. BMJ 2003; 327:1394-1395.

Artigo apresentado em 22/07/2007

Aprovado em 22/08/2007

Versão final apresentada em 14/09/2007 